\title{
ESCOLHA DE CANAIS DE VENDA EM COMÉRCIO ELET'RÔNICO
}

\section{CHOICE OF SALES CHANNELS IN ELECTRONIC COMMERCE}

\section{CLAUDIO SANTIAGO BAPTISTA}

Mestre em Gestão Empresarial pela Escola Brasileira de Administração Pública e de Empresas da Fundação Getulio Vargas (Ebape-FGV). Praia de Botafogo, 190/506 - Rio de Janeiro - RJ - CEP 22253-900 E-mail: ccssbb@terra.com.br

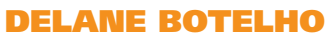

Doutor em Administração de Empresas pela Eaesp-FGV. Professor adjunto da Escola Brasileira de Administração Pública e de Empresas da Fundação Getulio Vargas (Ebape-FGV). Praia de Botafogo, 190/506 - Rio de Janeiro - RJ - CEP 22253-900 E-mail: dbotelho@fgr.br 


\section{RESUMO}

O comércio eletrônico no Brasil tem-se tornado um canal significativo de divulgação, comercialização e distribuição de produtos e serviços no que se refere a volumes físico e financeiro, porém existem muitas lacunas no conhecimento sobre o processo de escolha dos canais de venda no ambiente on-line. O objetivo deste trabalho é levantar e quantificar os atributos relevantes na escolha desses canais no contexto brasileiro. Na fase exploratória da pesquisa, foram realizadas entrevistas em profundidade com usuários de internet. A avaliação dos pesos dos atributos foi feita por um levantamento com compradores de livros de lojas de varejo eletrônico, usando a metodologia de análise conjunta. Os resultados mostraram que o preço foi o atributo mais importante, seguido de prazo de entrega, experiência positiva de compra anterior, usabilidade, indicação positiva de amigos e marca do site. São discutidas as implicações desses resultados para o marketing, além de sugestões para futuras pesquisas.

\section{PALAVRAS-CHAVE}

Escolha do consumidor; Análise conjunta; Comércio eletrônico.

\section{ABSTRACT}

Electronic commerce in Brazil has become a significant channel for the dissemination, commercialization and distribution of products and services in terms of physical and financial volume, however there are many gaps in knowledge about the process of choice of sales channels in the online environment. The objective of this work is to appraise and quantify the relevant attributes in the choice of these channels in the Brazilian context. In-depth interviews were conducted with Internet users during the exploratory phase of the research. The evaluation of the importance of these attributes was performed 
through a survey among purchasers of books in electronic retail stores, using conjoint analysis methodology. The results revealed that the price was the most important attribute, followed by delivery time, positive experience in prior purchases, usability, positive recommendation from friends and the brand name of the site. The implications of these results for marketing are then discussed and suggestions for future research are presented.

\section{KEYWORDS}

Customer choice; Conjoint analysis; Electronic commerce.

\section{INTRODUÇÃO}

O número de usuários domiciliares com acesso à internet no Brasil é de aproximadamente 2I,2 milhões, dos quais I3,2 milhões são considerados usuários ativos (INSTITUTO BRASILEIRO DE OPINIÃO PÚBLICA E ESTATÍSTICA, 2006). A Pesquisa Nacional por Amostra de Domicílios (Pnad) aponta que, em 2004, I6,3\% das residências no Brasil possuíam um microcomputador, dos quais I2,2\% com acesso à internet (INSTITUTO BRASILEIRO DE GEOGRAFIA E ESTATÍSTICA, 2006).

O valor transacionado por meio eletrônico entre empresas em 2005, no Brasil, representou I9,6\%, e as transações entre empresas e consumidores representaram $7,45 \%$ do total transacionado (FUNDAÇÃO GETULIO VARGAS, 2006). Essa transação eletrônica refere-se ao comércio eletrônico, entendido como a realização de comunicações e transações de negócios por meio de redes e computadores, mais especificamente a compra e a venda de produtos e serviços, e a transferência de fundos por comunicação digital (REEDY et al., 200I).

Comparado com o comércio tradicional, o processo de compra pela internet propicia uma facilidade de acesso e busca de produtos para o consumidor e, pelo lado do varejista, oferece um novo canal de distribuição e comunicação com os compradores. Este artigo analisa o ponto de vista do comprador, com implicações gerenciais para o varejista, pois tem o objetivo de levantar e quantificar os atributos relevantes na escolha, pelo comprador, dos canais de venda no varejo eletrônico de livros.

Existem hoje diversos estudos sobre atributos de escolha de produtos, canais de venda e apreçamento em comércio eletrônico, como os realizados por Talaga e Tucci (200I), Hernandez (2003), Aggarwal e Vaidyanathan (2003), Verma e 
Varma (2003) e Kung et al. (2002), mas muitos dos resultados encontrados por esses autores são divergentes, o que torna o tema ainda controverso. Em nenhum estudo da Academia Brasileira de Marketing, há tentativa de mensuração da relevância dos atributos de escolha dos canais de comércio eletrônico, o que motivou a realização deste estudo empírico.

O artigo está assim estruturado. Esta seção apresentou o objetivo e a relevância do tema. A próxima apresenta uma revisão da literatura relacionada às principais variáveis influenciadoras do processo de compra em comércio eletrônico. A terceira seção apresenta o método, que envolve tanto a pesquisa qualitativa como a quantitativa. A seguir são apresentados os resultados. O artigo é finalizado com a discussão das implicações de tais resultados para o marketing, com sugestões para futuras pesquisas.

\section{REVISÃO DE LITERATURA}

Nesta seção, são tratadas as variáveis/atributos que, pela literatura de marketing, são as mais relevantes como elementos influenciadores do processo de compra em comércio eletrônico.

A primeira variável refere-se ao preço. Segundo Clay et al. (200I) e Alba et al. (I997), a internet possibilita a busca de informações e comparação de preços e produtos com baixos custos, o que pode levar os consumidores à maior sensibilidade aos preços, implicando práticas de preços mais baixos no comércio virtual em comparação com o mundo físico.

Com base em dados coletados entre agosto de i999 e janeiro de 2000 e considerando o preço fixado para 399 livros por 32 livrarias on-line nos Estados Unidos, Clay et al. (200I) examinaram a estratégia de apreçamento desses varejistas e concluíram que mais competição conduz a preços mais baixos e a uma menor dispersão de preço. Além disso, os autores também observaram que os produtos mais extensamente anunciados tiveram também os preços mais baixos.

Alba et al. (1997) examinaram as implicações de compra via comércio eletrônico para consumidores, varejistas e fabricantes, e comprovaram que o desenvolvimento tecnológico oferece aos consumidores melhores oportunidades de localizar e comparar ofertas de produtos. Essa maior disponibilidade de informação em relação às características e aos preços dos produtos, aliada a uma maior variedade de produtos para a escolha, pode gerar uma tendência de declínio de preços ou uma busca de diferenciação no produto (SMITH et al., I999).

Os consumidores podem ser mais sensíveis ao preço quando compram pela internet, em razão do baixo custo de busca de informação on-line (HERNAN- 
DEZ, 2003), mas Nagle e Holden (2002) apresentam contra-argumentos para esse pressuposto: I. na internet os consumidores têm menos certeza sobre as características do produto a ser comprado, o que os torna menos dispostos a pagar preços mais baixos por alternativas de compra desconhecidas; 2. os consumidores têm menos certeza a respeito da legitimidade do varejista, o que os deixa dispostos a pagar mais para lidar com comerciantes mais respeitáveis e com maior legitimidade; 3. os compradores on-line têm maior renda, que é uma característica geralmente associada com consumidores menos sensíveis a preço; 4. pesquisas anteriores demonstraram que os compradores on-line usam a internet em busca de conveniência, e não de preços mais baixos.

A segunda variável é a confiança, que, na definição de Giffin (I967), ocorre quando uma pessoa: confia em outra, arrisca algo do valor e tenta alcançar um objetivo desejado. Há várias evidências empíricas sugerindo que uma das barreiras principais ao desenvolvimento do comércio eletrônico é a falta da confiança que existe entre varejistas e consumidores eletrônicos (GUPTA e CHATTERJEE, I997; HOFFMAN et al., I998; JARVENPAA e TODD, I997; URBAN et al., 2000).

Urban et al. (2000) afirmam que os consumidores tomam decisões de compra na internet com base na confiança, e a "quantidade de confiança" que um site necessita passar para o consumidor depende da categoria do produto, da pressão competitiva dos novos intermediários, da informação disponível e da capacidade de inovação do site. A confiança pode ter sua importância aumentada em mercados eletrônicos por causa das separações espacial (física) e temporal entre os compradores e os vendedores, as quais são inerentes ao comércio eletrônico (BRYNJOLFSSON e SMITH, 2000). É esperado que o varejo eletrônico cresça a taxas agressivas em anos futuros, mas uma possível falta de confiança dos clientes em potencial pode impedir esse crescimento.

Outra variável é a marca. Mesmo para os consumidores que utilizam ferramentas de busca e comparação de preços de produtos em oferta on-line, que provavelmente seriam aqueles mais sensíveis a preço, a marca do varejista parece indicar um sinal de qualidade de serviços. Brynjolfsson e Smith (200I) afirmam que os consumidores usam a marca como uma proxy para a credibilidade do varejista em aspectos não contratuais do produto e da prestação de serviços, tal como a confiabilidade do transporte. Num estudo com compradores de livros em livrarias virtuais, Kung et al. (2002) observaram que os compradores propunham-se a pagar mais por livros comprados em livrarias mais conhecidas. A marca, portanto, foi um atributo determinante da escolha do consumidor. Tais autores afirmam ainda que, por causa das preocupações com segurança, compradores on-line tendem a ter lealdade de marca para com certos sites onde tiveram experiências positivas. 
Desenvolver reputação de marca e cobrar preços premium pelos produtos é uma estratégia já há muito existente no mundo não virtual (LATCOVICH e SMITH, 200I). Latcovich e Smith (200I) concluíram que marca, visibilidade e confiança permanecem como fontes importantes da diferenciação entre varejistas na internet.

A usabilidade do site, que também pode ser uma variável relevante, inclui facilidade de uso, atualização freqüente de conteúdo, rapidez (tempo mínimo de resposta(download), relevância para os usuários e conteúdo de alta qualidade (NIELSEN, I993). A International Standards Organization (I994) define usabilidade como "até que ponto um produto pode ser usado por usuários-alvo para alcançar metas específicas com efetividade, eficiência e satisfação em um contexto especificado de uso".

No comércio eletrônico, é inerente ao processo de compra a interação (uso) do consumidor com o site ofertante. Essa interação tem sido analisada por diversos autores no que se refere à usabilidade. Heijden (200I) examinou a usabilidade e a satisfação de uso considerando seus impactos na intenção de uso e no uso real dos portais da internet, com uma amostra de compradores on-line, e concluiu que esses fatores influenciam diretamente na escolha dos sites. Ou seja, sites com atratividade visual e fáceis de usar estimulam positivamente o seu uso.

Delleart e Kahn (I999) observaram que sites mais lentos tendem a ser avaliados negativamente pelos consumidores. Nielsen (I999) afirma que o tempo de carregamento das páginas da internet pode causar impactos na usabilidade e afetar seus usuários de dois modos possivelmente relacionados: I. com a demora no carregamento das páginas, a atenção do usuário não permanecerá focalizada na tarefa de recuperação de informação, e ele pode dividir sua atenção entre essa tarefa e outras relacionadas ou não com o computador; 2 . os usuários podem se frustrar com demoras imprevisíveis e, por isso, desistir da tarefa de recuperação de informação, abandonando o site.

A usabilidade pode estar relacionada à confiança. A qualidade da interface do usuário no site pode ser determinante no estabelecimento inicial de confiança. Roy et al. (200I) descrevem um estudo em que 66 consumidores foram solicitados a executar algumas tarefas predefinidas de compra em uma série de locais com qualidade de interface variada. Como resultado, foi encontrada uma relação forte entre qualidade da interface e confiança.

O modelo proposto neste artigo tem por objetivo explicar a escolha do canal de venda de livros via comércio eletrônico por consumidores e a importância relativa de cada uma das variáveis levantadas nesta seção (e outras abordadas na fase exploratória da pesquisa, descrita adiante). A próxima seção apresenta o método de pesquisa utilizado. 


\section{MÉTODO}

Para a operacionalização deste estudo em comércio eletrônico, foi escolhido o setor de livros, o que propicia algumas vantagens para a quantificação dos atributos, como: I. definição mais precisa para o comprador ao se especificar um título de livro a ser avaliado (no caso desta pesquisa, um dicionário específico); 2. essa definição colabora para minimizar o efeito de variáveis intervenientes do produto, como marca, embalagem e garantia, possibilitando maior foco nas variáveis de escolha do canal (de interesse deste artigo); 3. é mais fácil obter consumidores experientes na compra de livros via comércio eletrônico. Abordagem semelhante foi adotada por Talaga e Tucci (200I).

As variáveis influenciadoras da escolha, analisadas no referencial teórico, foram complementadas ou confirmadas na fase exploratória da pesquisa. Uma vez que, para a adoção do modelo de análise conjunta (explicado adiante), é necessário que todas as variáveis sejam previamente escolhidas (MALHOTRA, 200I), realizou-se uma pesquisa qualitativa para indicar que outros atributos seriam relevantes no processo decisório de compra em comércio eletrônico, para pessoas do perfil da amostra analisada, além daqueles obtidos na literatura anteriormente apresentada.

A etapa qualitativa foi usada na compreensão das variáveis (atributos) relevantes a serem consideradas (AAKER et al., I997). Foram feitas entrevistas em profundidade com oito homens e mulheres, maiores de $2 \mathrm{I}$ anos, residentes na cidade do Rio de Janeiro e compradores habituais via comércio eletrônico, os quais possuíam experiência na compra de livros on-line. Para a elaboração do roteiro de entrevista, consideraram-se as variáveis obtidas no estudo do referencial teórico, a fim de abordar variáveis que poderiam influenciar a escolha: produto a ser comprado, preço, confiança no processo de compra on-line, marca e usabilidade dos sites. Perguntas de respostas espontâneas também foram introduzidas no roteiro. Consideraram-se também as variáveis "processo de entrega do produto" e "promoção" (divulgação do site e dos produtos), as quais foram obtidas nas entrevistas iniciais. O roteiro de entrevistas foi, então, enriquecido no decorrer das duas primeiras entrevistas, que funcionaram como um teste, na busca de identificar e eliminar problemas e complementar os tópicos abordados (MALHOTRA, 200I).

Após a análise e comparação, tanto das gravações quanto das transcrições das oito entrevistas, identificaram-se os atributos considerados pelos entrevistados como principais na escolha de livrarias on-line, e houve alta convergência de respostas espontâneas sobre tais atributos. Os principais foram: a) preço total (produto + frete); b) prazo de entrega do produto; c) conhecimento do site (marca); 
d) experiência anterior positiva de compra no site; e) indicação positiva de amigos para compra no site; f) usabilidade do site (principalmente tempo de resposta).

De posse desses atributos, passou-se para a fase quantitativa da pesquisa, em que se utilizou a análise conjunta que se aplica para a avaliação de escolhas de consumidores. Segundo Malhotra (200I), trata-se de uma técnica que procura determinar a importância relativa que os consumidores dão a atributos relevantes e à utilidade que eles associam aos níveis de atributos, em que os entrevistados deparam com estímulos que consistem em combinações de níveis de atributos, e devem analisar tais estímulos em função de sua conveniência. Tal análise procura estabelecer funções de valor que descrevem o grau de utilidade que os consumidores associam aos níveis de cada atributo, ou seja, provê uma medida quantitativa da importância relativa de um atributo em relação a outro (AAKER et al., I997).

A análise conjunta aborda uma questão central na formulação de estratégias: o motivo pelo qual os consumidores escolhem uma marca ou um fornecedor de serviço em detrimento de outro. A idéia central é que produtos ou serviços podem ser decompostos em um conjunto de atributos (GREEN, I999).

A variável dependente é o julgamento de preferência que o respondente faz sobre um determinado conceito, e as variáveis independentes são os níveis dos atributos que são avaliados dois a dois ou em perfis (contendo todos os atributos). Um valor de utilidade relativa é associado a cada nível dos atributos, e a premissa básica é a de que as pessoas avaliam conceitos pelo somatório das avaliações dos atributos individuais associados ao conceito (AAKER et al., I997). O modelo matemático (LOUVIERE, I998) é assim descrito:

$$
\mathrm{Y}=\beta_{\mathrm{I}} * \text { Atributo I }+\beta_{2} * \text { Atributo } 2+\beta_{3} * \text { Atributo } 3+\ldots+\beta_{\mathrm{n}} * \text { Atributo } \mathrm{n}+\varepsilon
$$

em que $Y=$ escolha do canal de venda; $\beta_{\mathrm{I}}$ a $\beta_{\mathrm{n}}=$ importância relativa dos atributos; $\varepsilon=$ erro do modelo. Nesse caso, quanto maior for o valor de $\beta$, maior será a importância (influência ou peso) da variável (atributo) na escolha do canal.

Um levantamento foi realizado com a distribuição de questionários estruturados. Tais questionários continham uma pergunta-filtro, com o objetivo de eliminar todos os respondentes que não tivessem realizado nenhuma compra on-line nos seis meses anteriores ao levantamento, a fim de concentrar a amostra nos compradores regulares de comércio eletrônico. Foram também incluídas perguntas para captar características demográficas dos respondentes.

Os questionários foram distribuídos para os e-mails pessoais de possíveis respondentes - homens e mulheres, maiores de 18 anos, residentes no Brasil, 
principalmente na cidade do Rio de Janeiro, compradores habituais via comércio eletrônico, que possuíam experiência na compra de livros on-line. Aplicaram-se também questionários impressos para alunos de cursos de graduação e pós-graduação de três importantes universidades da cidade do Rio de Janeiro. A amostra foi por conveniência, não probabilística, o que impossibilita representatividade em relação a qualquer população definível. Como resposta, obtiveram-se I6I questionários respondidos, sendo 73 via e-mail e 88 em meio físico (impresso). Desse total, I22 são válidos para uso neste estudo, pois provêm de respondentes que realizaram, no mínimo, uma compra virtual nos últimos seis meses.

Adotou-se a metodologia tradicional de análise conjunta, ou seja, com um número limitado de atributos, dado que a relevância geral dos atributos foi obtida no estudo qualitativo. Utilizou-se também o método de escolha de perfis que, segundo Hair (1998), presta-se bem para uma pesquisa que tenha até nove atributos, permitindo um nível de análise individual (nível de respondente). A limitação é o pequeno número de atributos que pode ser realisticamente avaliado por um respondente (AAKER et al., I997).

\section{QUADRO I}

ATRIBUTOS E SEUS NÍVEIS

\begin{tabular}{ll}
\hline ATRIBUTO & NÍvEIS \\
\hline Conhecimento do site (marca) & $\begin{array}{l}\text { Livraria Saraiva (marca conhecida) } \\
\text { Livraria Sodiler (marca desconhecida) }\end{array}$ \\
\hline Preço total (produto + frete) & $\begin{array}{l}\mathrm{R} \$ 147,00 \\
\mathrm{R} \$ 189,00\end{array}$ \\
\hline Prazo de entrega do livro & Dois dias úteis \\
\hline Experiência anterior positiva de & Sim úteis \\
compra no site & Não \\
\hline Indicação positiva de amigos para & Sim \\
compra no site & Não \\
\hline & O site é muito rápido e fácil - é possível fechar a compra \\
em menos de três minutos. \\
Usabilidade do site
\end{tabular}


As variáveis mencionadas na etapa exploratória formaram os atributos mostrados no Quadro I. Realizaram-se pré-testes do questionário com dez respondentes, com resultados muito importantes para o prosseguimento da pesquisa, a saber:

I. Necessidade de especificar um livro (título) a ser usado como objeto de compra, pois, nas diversas formas testadas em que o respondente trabalhava com um título hipotético, surgiam discussões sobre o produto a ser comprado, tirando o foco da análise da escolha do canal de venda. O exemplo do Dicionário de língua portuguesa Aurélio foi bem-aceito por todos e, portanto, adotado aqui.

2. A escolha do livro mencionado no item anterior orientou a escolha dos níveis de preços a serem testados. Os valores de R\$ I47,00 e R\$ I89, ०0 estão dentro de um intervalo de preços realmente praticado no varejo eletrônico durante o período de coleta de dados deste trabalho.

3. Necessidade de usar duas marcas reais de sites, pois o uso de um site hipotético tornava o exercício muito distante da situação real de compra dos respondentes. A fim de resolver esse problema, foram usados os sites (marcas) da Livraria Saraiva e da Livraria Sodiler. Segundo o site de pesquisa em comércio eletrônico e-bit, o site da Livraria Saraiva, de agosto de 2000 a dezembro de 2005 , foi avaliado I96.506 vezes por seus consumidores, seja no ato da compra seja no pós-venda. O site da Livraria Sodiler, pelos mesmos critérios, foi avaliado I8.050 vezes. Esse foi o parâmetro usado para balizar o nível de conhecimento do site.

4. No caso do prazo de entrega, foi necessário ampliar a diferença entre o prazo mais curto e o mais longo. Inicialmente haviam sido usados prazos de dois e cinco dias úteis. Com os resultados iniciais, os prazos passaram para dois e dez dias úteis, pois a diferença inicial era considerada pouco relevante pelos respondentes.

5. De forma análoga, no caso do tempo total para fechar a compra, foi necessário ampliar a diferença entre o prazo mais curto e o mais longo. Inicialmente haviam sido usados prazos de menos de três e mais de cinco minutos. Com os resultados iniciais, os prazos passaram para menos de três e mais de dez minutos, pois a diferença inicial também foi considerada pouco relevante pelos respondentes.

6. Finalmente, foram necessários alguns ajustes nos textos explicativos e enunciados das questões formuladas, a fim de facilitar o entendimento e o correto preenchimento do questionário. 
No Quadro I, verifica-se que existem seis atributos, com dois níveis (valores) para cada um deles. Esse quadro possibilita calcular o número de perfis (estímulos) que deverão ser analisados pelos respondentes.

A escolha do método de perfil completo - composto por um nível de cada um dos atributos avaliados, como metodologia de apresentação dos estímulos - baseia-se na afirmação de Hair (I998) acerca das vantagens desse método sobre os demais: I. descrição mais realista do estímulo, contendo um nível para cada fator; 2. retrato mais realista do processo de escolha entre os fatores e das correlações ambientais existentes entre os atributos; e 3. possibilidade de uso de diferentes julgamentos de preferência, como intenção de compra, por exemplo, que é difícil de ser respondida em outros métodos.

Dessa forma, cada perfil (estímulo) é um conjunto de combinações dos diversos valores (níveis) de cada atributo. Incluindo seis atributos com dois níveis cada, tem-se o seguinte desenho fatorial: $2^{6}=6_{4}$ estímulos (perfis), a serem analisados pelos respondentes. Adotou-se o desenho fatorial fracionado criando um subconjunto ortogonal de estímulos (não há correlação entre os estímulos) e balanceado (cada nível de um atributo/fator aparece igual número de vezes). A redução ortogonal gerou oito perfis para avaliação dos atributos e seus níveis, além de quatro estímulos adicionais para validação do modelo (após a coleta e processamento dos dados quantitativos obtidos dos respondentes). A Tabela I apresenta os estímulos.

O conjunto de estímulos, apresentados na Tabela I, foi apresentado aos respondentes em quatro diferentes modelos de questionários. A única diferença entre os quatro modelos foi a ordenação dos estímulos, visto que a ordem em que os estímulos são listados para o respondente pode ter impacto na sua avaliação (HAIR, I998).

Para a avaliação (escolha) dos perfis apresentados pelos respondentes, adotou-se uma escala Likert de cinco pontos, em que o valor "I" corresponde a "dificilmente compraria" e o valor "5", a "certamente compraria" 


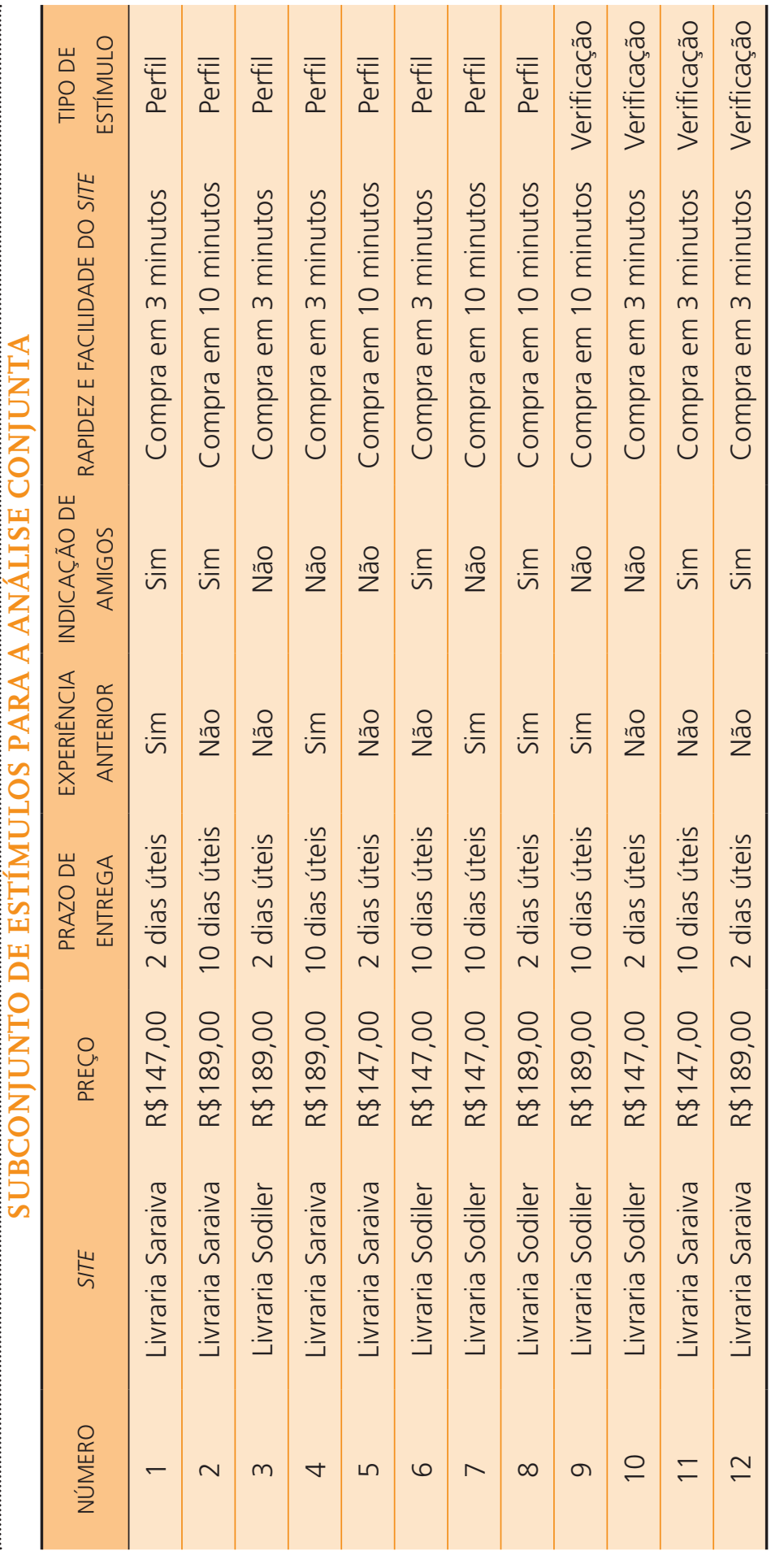




\section{RESULTADOS}

Na Tabela 2, são apresentados os valores das importâncias médias de cada atributo, bem como o fator de utilidade para cada nível de cada um dos atributos. O Gráfico I ilustra as importâncias dos atributos.

\section{TABELA 2}

VALORES DA IMPORTÂNCIA DOS ATRIBUTOS, UTILIDADE DOS NÍVEIS DOS ATRIBUTOS E CONSTANTE DO MODELO

\begin{tabular}{|c|c|c|c|}
\hline ATRIBUTO & IMPORTÂNCIA MÉDIA & NÍVEL & UTILIDADE \\
\hline \multirow{2}{*}{ Preço } & \multirow{2}{*}{$28,58 \%$} & $\mathrm{R} \$ 147,00$ & $-4,0020$ \\
\hline & & $\mathrm{R} \$ 189,00$ & $-5,1455$ \\
\hline \multirow{2}{*}{ Prazo de entrega } & \multirow{2}{*}{$22,27 \%$} & 2 dias úteis & $-0,2100$ \\
\hline & & 10 dias úteis & $-1,0502$ \\
\hline \multirow{2}{*}{$\begin{array}{l}\text { Experiência positiva } \\
\text { de compra anterior }\end{array}$} & \multirow{2}{*}{$14,47 \%$} & Sim & $-0,5451$ \\
\hline & & Não & $-1,0902$ \\
\hline \multirow{2}{*}{$\begin{array}{l}\text { Usabilidade do } \\
\text { site }\end{array}$} & \multirow{2}{*}{$12,48 \%$} & $\begin{array}{c}\text { Compra em menos de } \\
3 \text { minutos }\end{array}$ & $-0,1022$ \\
\hline & & $\begin{array}{c}\text { Compra em mais de } 10 \\
\text { minutos }\end{array}$ & $-0,3408$ \\
\hline \multirow{2}{*}{$\begin{array}{l}\text { Indicação positiva } \\
\text { de amigos }\end{array}$} & \multirow{2}{*}{$11,95 \%$} & Sim & $-0,3852$ \\
\hline & & Não & $-0,7705$ \\
\hline \multirow{2}{*}{ Site (marca) } & \multirow{2}{*}{$10,25 \%$} & Livraria Saraiva & $-0,2049$ \\
\hline & & Livraria Sodiler & $-0,4098$ \\
\hline Constante do Model & $: 10,0563$ & & \\
\hline
\end{tabular}

Com base nesses resultados, é possível destacar três grupos de importância de atributos. No primeiro, estão o preço com um peso relativo de $28,58 \%$ e o prazo de entrega com $22,27 \%$. Esses dois atributos, elementos do composto mercadológico, respondem por mais de $50 \%$ de influência sobre a escolha do canal de venda em comércio eletrônico, neste estudo. $\mathrm{O}$ terceiro atributo mais 
importante foi a experiência positiva de compra anterior (I4,47\%), e, se for somado com o quinto atributo mais valorizado (indicação positiva de amigos, II,95\%), tem-se um peso relativo de $26,42 \%$ para os atributos relacionados à questão da confiança. Portanto, somando-se a importância dos dois atributos relacionados à confiança, esta se torna o segundo fator mais importante para a decisão relativa na escolha do site.

\section{TABELA 3}

IMPORTANCIA RELATIVA DOS ATRIBUTOS PARA ESCOLHA DO CANAL DE VENDA EM COMÉRCIO ELETRÔNICO

\begin{tabular}{|c|c|c|c|}
\hline ATRIBUTO & IMPORTÂNCIA MÉDIA & NÍVEL & UTILIDADE \\
\hline \multirow{2}{*}{ Preço } & \multirow{2}{*}{$28,58 \%$} & $R \$ 147,00$ & $-4,0020$ \\
\hline & & $\mathrm{R} \$ 189,00$ & $-5,1455$ \\
\hline \multirow{2}{*}{ Prazo de entrega } & \multirow{2}{*}{$22,27 \%$} & 2 dias úteis & $-0,2100$ \\
\hline & & 10 dias úteis & $-1,0502$ \\
\hline \multirow{2}{*}{$\begin{array}{l}\text { Experiência positiva de } \\
\text { compra anterior }\end{array}$} & \multirow{2}{*}{$14,47 \%$} & Sim & $-0,5451$ \\
\hline & & Não & $-1,0902$ \\
\hline \multirow{2}{*}{ Usabilidade do site } & \multirow{2}{*}{$12,48 \%$} & $\begin{array}{l}\text { Compra em menos de } \\
3 \text { minutos }\end{array}$ & $-0,1022$ \\
\hline & & $\begin{array}{l}\text { Compra em mais de } \\
10 \text { minutos }\end{array}$ & $-0,3408$ \\
\hline \multirow{2}{*}{$\begin{array}{l}\text { Indicação positiva de } \\
\text { amigos }\end{array}$} & \multirow{2}{*}{$11,95 \%$} & Sim & $-0,3852$ \\
\hline & & Não & $-0,7705$ \\
\hline \multirow{2}{*}{ Site (marca) } & \multirow{2}{*}{$10,25 \%$} & Livraria Saraiva & $-0,2049$ \\
\hline & & Livraria Sodiler & $-0,4098$ \\
\hline \multicolumn{4}{|c|}{ Constante do Modelo: 10,0563 } \\
\hline
\end{tabular}

O quarto atributo mais importante é a usabilidade (I2,48\%) e o último é a marca do site (importância relativa de io,25\%). Este é o terceiro grupo, com os atributos mais diretamente relacionados ao site em si, somando 22,73\% de importância.

Com relação à validação estatística do modelo, os coeficientes $R$ de Pearson $(0,986 ; p=0,0000)$ e Tau de Kendall (0,857; $p=0,0015)$ indicaram haver uma 
alta correlação entre as preferências observadas (respostas dos entrevistados) e estimadas (calculadas pelo modelo).

O modelo aqui obtido, pela análise conjunta, de escolha do canal de venda de livros via comércio eletrônico é:

$$
\begin{aligned}
\mathrm{Y}= & 0,2858 * \text { Preço } \\
& +0,2227 * \text { Prazo de entrega } \\
& +0, \mathrm{I} 447 * \text { Experiência positiva de compra anterior } \\
& +0, \mathrm{I} 248 * \text { Usabilidade do site } \\
& +0, \mathrm{II} 95 * \text { Indicação positiva de amigos } \\
& +0, \mathrm{IO} 25 * \text { Marca do site } \\
& +\varepsilon
\end{aligned}
$$

A seção seguinte discute os resultados aqui apresentados e suas implicações gerenciais e para o marketing.

\section{CONSIDERAÇÕES FINAIS}

Os resultados deste estudo parecem evidenciar a importância do atributo preço, reforçando a afirmação de que a possibilidade de busca de informações e comparação de preços e produtos na internet, com baixos custos, levaria os consumidores a uma maior sensibilidade a preços (CLAY et al., 200I; ALBA et al., I997).

$\mathrm{O}$ segundo fator mais valorizado pelos respondentes refere-se ao prazo de entrega, que surgiu na pesquisa qualitativa, pois todos os entrevistados na fase exploratória mencionaram de forma espontânea esse atributo como importante na avaliação final da opção de compra. No referencial teórico, entretanto, essa variável não surgiu como um atributo muito relevante. Isso pode indicar novas atenções para essa variável no Brasil, indicando diferenças nacionais em relação ao comportamento do consumidor americano ou europeu, o que significa possibilidades para futuras pesquisas.

O terceiro atributo mais importante na amostra analisada seria a "experiência positiva de compra anterior", que representa a variável confiança. Realmente, de acordo com Brynjolfsson e Smith (2000), uma das barreiras principais ao desenvolvimento do comércio eletrônico é a falta da confiança que existe entre varejistas e consumidores eletrônicos. Com base nisso, a confiança poderia ser um dos componentes mais importantes de um programa de marketing eficaz para internet. O presente estudo confirma a importância desse atributo na amostra analisada. 
A usabilidade do site, que aqui foi avaliada no que se refere à sua rapidez e facilidade de uso, tangibilizada pelo tempo necessário para concluir um processo de compra, foi o quarto atributo mais influente da escolha. Tanto os resultados qualitativos quanto os quantitativos parecem reforçar a importância da usabilidade dos sites em comércio eletrônico, tanto no que diz respeito à facilidade de uso quanto ao tempo de resposta.

A preponderância do terceiro atributo sobre o quinto ("indicação de amigos", também ligado ao atributo confiança) parece um tanto óbvia, dado que o terceiro trata de uma percepção adquirida por experiência própria e o quinto de uma percepção construída com base em referências de terceiros, o que naturalmente torna este menos confiável.

Por último, desponta a marca do site. Isso não foi um resultado esperado, em razão de toda a importância acadêmica e gerencial que é dada ao estudo de marcas; bem como todo o investimento feito pelas empresas de comércio eletrônico na construção do conhecimento de suas marcas em relação ao público consumidor. Talvez a operacionalização dessa variável neste estudo, com apenas duas marcas, possa ter tido alguma influência (estudos futuros poderiam testar mais de duas marcas).

Este trabalho vem se somar aos estudos já existentes sobre o comércio eletrônico, como Hernandez (2003) e Costa (2004), trazendo, pela primeira vez no Brasil, a aplicação de análise conjunta na escolha do canal. Parte significativa do referencial teórico existente sobre o assunto vem de estudos realizados em outros países, não existindo, em comparação, produção acadêmica consistente sobre o tema. Implicações gerenciais dizem respeito à tomada de decisão pelos varejistas em comércio eletrônico, com relação aos principais atributos que devem ser trabalhados por eles com relação ao canal de vendas eletrônico, a fim de incrementar a probabilidade de escolha de suas lojas virtuais pelos consumidores na internet. Sabendo-se dos atributos mais valorizados, e quanto eles são valorizados, os varejistas do comércio eletrônico podem direcionar seus esforços e recursos de forma mais focalizada, a fim de melhorar seus resultados de vendas pela internet.

Trabalhar principalmente preços competitivos bastante visíveis no site e prazos de entregas reduzidos, incentivando a primeira compra e as compras repetidas, além de tornar os sites rápidos e fáceis de navegar, estimulando a indicação de compras entre amigos e divulgando a marca dos sites, parece ser um conjunto de esforços que tende a aumentar a concretização de vendas, pelo menos no setor de livraria virtual. Obviamente, tais atributos poderiam ser empregados num futuro estudo, com a utilização de uma amostra probabilística.

Com este artigo, podem-se abrir possibilidades para novos estudos de análise conjunta, considerando outros tipos de produtos que tenham atributos di- 
ferentes dos abordados aqui - preço, público-alvo, freqüência de compra e nível de concorrência, entre outros. Uma das limitações deste estudo se refere ao fato de terem sido adotados somente dois níveis de preços com diferença expressiva entre ambos - o menor preço é $22 \%$ mais baixo do que o maior, o que pode ter aumentado a relevância desse atributo. Isso também pode ser verificado em estudos similares. Outra abordagem interessante seria analisar e comparar os estudos existentes sobre os atributos mais relevantes para o varejo eletrônico, buscando identificar alguma possível relação entre a importância do atributo e o tipo de produto, caso exista.

\section{REFERÊNCIAS}

AAKER, D. A.; KUMAR, V.; DAY, G. S. Marketing research. 6. ed. John Wiley \& Sons Inc., I997. AGGARWAL P.; VAIDYANATHAN, R. Eliciting online customers preferences: conjoint vs selfexplicated attribute-level measurements. Journal of Marketing Management, v. I9, p. I57-I77, 2003. BRYNJOLFSSON, E.; SMITH, M. Frictionless commerce? A comparison of internet and conventional retailers. Management Science, Linthicum, v. 46, n. 4, p. 563-585, Apr. 2000.

CLAY et al. Price and price dispersion on the web: evidence from the online book industry. The Journal of Industrial Economics, v. 49, n. 4, p. 52I-54I, $200 \mathrm{I}$.

COSTA, F. C. X. Influências ambientais e o comportamento de compra por impulso: um estudo em lojas físicas e virtuais. 2003. Tese (Doutorado) - Faculdade de Economia, Administração e Contabilidade, Universidade de São Paulo, São Paulo, 2003. Disponível em: <http://www.teste.usp.br>. Acesso em: 18 jul. 2004.

FUNDAÇÃO GETULIO VARGAS. Pesquisa anual CIA. Disponível em: <http://www.eaesp.fgvsp. br/subportais/Interna/Relacionad/FGV2006Pesı2.pdf>. Acesso em: II abr. 2006.

GIFFIN, K. The contributions of studies of source credibility to a theory of interpersonal trust in the communication process. Psychological Bulletin, n. 68, p. I04-I20, I967.

GREEN, P. E.; KRIEGER, A. M. Usando a análise conjunta para ver a interação competitiva com os olhos do cliente. In: DAY, G. S.; REIBSTEIN, D. J. A dinâmica da estratégia competitiva. Rio de Janeiro: Campus, I999.

GUPTA, S.; CHATTERJEE, R. Consumer and corporate adoption of the world wide web as a commercial medium. In: PETERSON, R. A. (Ed.). Electronic marketing and the consumer. California: I997. p. I23-I38.

HAIR, J. F. et al. Multivariate data analysis. 5. ed. Prentice Hall, I998.

HEIJDEN, H. van der. Factors influencing the usage of websites: the case of a Generic Portal in Netherlands. e-Everything: e-Commerce, e-Government, e-Household, e-Democracy. In: $\mathrm{I}^{\mathrm{TH}}$ BLED ELETRONICS COMMERCE CONFERENCE. Bled, Slovenia, Jun. 25-25 200I.

HERNANDEZ, J. M. da C. Price sensitivity on the internet: the role of trust in the retailer. Enanpad, 2003.

HOFFMAN, D. L.; NOVAK, T.P.; CHATTERJEE, P. Commercial scenarios for the web: opportunities and challenges. Journal of Computer-Mediated Communication, v. I, n. 3, I998. 
INSTITUTO BRASILEIRO DE GEOGRAFIA E ESTATÍSTICA. Pesquisa Nacional de Amostragem por Domicílio 2004. Teleco. Disponível em: <http://www.teleco.com.br/internet.asp >. Acesso em: I6 jul. 2006.

INSTITUTO BRASILEIRO DE OPINIÃO PÚBLICA E ESTATÍSTICA. Comércio online lidera crescimento da web brasileira em abril. Disponível em: <http://www.ibope.com.br>. Acesso em: i6 jul. 2006.

INTERNATIONAL STANDARDS ORGANIZATION (ISO). Ergonomic requirements for office work with visual display terminals. Part II: Guidance on usability (ISO DIS 924I-II). London, I994. JARVENPAA, S. L.; TODD, P. A. Is there a future for retailing on the internet. In: PETERSON, R. A. (Ed.). Electronic marketing and the consumer. California: I997. p. I39-I54.

KUNG, M.; KENT, B. M.; COX, J. L. Pricing on the internet. Journal of Product \& Brand Management, v. II, n. 5, p. 274-287, 2002.

LATCOVICH, S.; SMITH, H. Pricing, sunk costs and market structure online: evidence from book retailing. Oxford Review of Economic Policy, v. I7, p. 217-234, $200 \mathrm{I}$.

LOUVIERE, J. J. Analyzing decision making: metric conjoint analysis. Newbury, Park, CA: Sage Publication, I998.

MALHOTRA, N. Pesquisa de marketing: uma orientação aplicada. 3. ed. Porto Alegre: Bookman, 200I. McCARTHY, E. J. Basic marketing: a managerial approach. I2. ed. Homewood: Irwin, I996.

NAGLE, T.; HOLDEN, R. K. The strategy and tactics of pricing: a guide to profitable decision making. Upper Saddle River, NJ: Prentice-Hall, 2002.

NIELSEN, J. Usability engineering. New York: Morgan Kaufmann, I993. Designing web usability. Indianapolis, IN: New Riders, I999.

REEDY, J.; SCHULLO, S.; ZIMMERMAN, K. Marketing eletrônico: da integração dos recursos eletrônicos ao processo de marketing. Porto Alegre: Bookman, 200I.

ROY, M. C.; DEWIT, O.; AUBERT, B. The impact of interface usability on trust in web retailers. Internet Research, Bradford, v.II, n. 5, p. 388-398, $200 \mathrm{I}$.

SMITH, M. D.; BAILEY, J.; BRYNJOLFSSON, E. Understanding digital markets: review and assessment. Disponível em: <http://www.e-commerce.mit.edu/papers/ude>. Acesso em: 20 jul. 2005 .

TALAGA, J. A.; TUCCI, L. A. Journal of Consumer Marketing, v. I8, n. I, p. I0-20, 200 I.

URBAN, G. L.; SULTAN, F.; QUALLS, W. J. Placing trust at the center of your internet strategy. Sloan Management Review, v. 42, n. I, p. 39-48, 2000.

VERMA, D. P. S.; VARMA, G. Online pricing: concept, methods and current practices. Journal of Services Research, v. 3, n. I, 2003.

TRAMITAÇÃO

Recebido em 13/3/2006

Aprovado em 18/7/2006 
Copyright of Revista de Administração Mackenzie is the property of Universidade Presbiteriana Mackenzie, RAM-Revista de Administracao Mackenzie and its content may not be copied or emailed to multiple sites or posted to a listserv without the copyright holder's express written permission. However, users may print, download, or email articles for individual use. 